\title{
Éléments du parcours institutionnel régional
}

\author{
Marc-Urbain Proulx ${ }^{1}$ \\ Université du Québec à Chicoutimi
}

A u cours de la deuxième moitié du $\mathrm{xx}^{\mathrm{e}}$ siècle, le paysage de la région du Saguenay-LacSaint-Jean s'est considérablement modifié à la faveur de la politique gouvernementale. Le résultat le plus marquant à propos du nouveau paysage régional réside dans un aménagement extensif du territoire. À cet effet d'aménagement, les acquis passés en matière de bâtiments collectifs (églises, hôtels de ville, écoles, hôpitaux...), de barrages, d'aqueducs, de routes, des ponts, des chemins, etc. furent substantiellement bonifiés par un vaste programme public de construction physique de ports, de collèges, d'aéroports, d'autoroutes, de réseaux électriques, de polyvalentes, de centres sportifs, d'édifices administratifs, de centres communautaires, etc. Selon cette approche interventionniste de nature physico-spatiale, divers services publics (entretien, conservation, protection, conseils...) furent aussi mis en œuvre dans les secteurs des ressources naturelles, de l'agriculture, de la faune, du récréotourisme, de la culture...

Pour gérer ces nouveaux équipements, infrastructures et services structurants pour le territoire du Saguenay-Lac-Saint-Jean, des organisations formelles furent mises en place afin de seconder les corporations municipales et les commissions scolaires déjà présentes. Directions régionales, conseils régionaux, centres, corporations, bureaux, offices, associations, sociétés, etc. se sont ainsi multipliés sur le terrain afin de relever des missions publiques spécifiques. En réalité, une véritable construction institutionnelle territoriale fut effectuée à deux nouveaux échelons distincts ${ }^{2}$. D’abord, à l'échelle de la région administrative ${ }^{3}$ du Saguenay-Lac-Saint-Jean au cours des années 1970, le mouvement qui avait commencé bien avant s'est intensifié pour ensuite se poursuivre lentement pendant les années 1980. Ensuite, les quatre territoires
MRC ont subi le même envol institutionnel à partir de 1980 pour s’accélérer au cours de la décennie 1990.

À l'apogée de cette période de construction physique et institutionnelle, les impulsions endogènes du développement se sont inscrites telle une véritable stratégie poursuivie par les gouvernements supérieurs. On a alors cherché à construire une nouvelle capacité de développement culturel, social et économique en misant sur des mécanismes locaux et régionaux de soutien à l'innovation. Les travailleurs et les entrepreneurs sont ainsi devenus des clients privilégiés des programmes gouvernementaux relativement généreux pour appuyer les diverses initiatives dites par le bas ou ascendantes. Formation professionnelle, $R \& D$, incubation industrielle, financement du risque, transferts technologiques, services aux entreprises, etc. représentent maintenant les nouvelles cibles des interventions publiques. Tant et si bien que le Saguenay-Lac-SaintJean dispose désormais des composantes de base d'une assise territoriale d'innovation et de production à transformer en un véritable système fertile, notamment par la création collective desdites « économies de proximité », étape ultime de la construction territoriale.

Cette construction territoriale multidimensionnelle à l'œuvre en région 02 au cours des dernières décennies est associée à un "chemin parcouru » relativement riche sous l'angle de la dotation d'un vaste domaine public apte à soutenir non seulement ses clients (population, travailleurs et entreprises), mais aussi l'appropriation collective de leviers du développement. L’imbrication régionale globale de ce «soutien territorial " représente certes une finalité à poursuivre selon des principes tels que la cohérence administrative, l'efficacité économique, la démocratie, la 
justice sociale et la durabilité du développement. La planification territoriale s'inscrit, à cet effet, telle la démarche collective privilégiée pour donner sens et moyens à ce régionalisme souhaité sous la forme de diverses actions structurantes.

Or, face à la turbulence très actuelle ${ }^{4}$ causée par une importante restructuration économique et sociale vécue à la faveur de ladite mondialisation et son corollaire, la métropolisation, notre question de recherche concerne la capacité d'action territoriale réelle face à la nécessité de repositionnement, voire de rebondissement de la région Saguenay-Lac-Saint-Jean. Dans ce texte, nous tenterons d'éclairer cette question en utilisant une grille de lecture institutionnelle ${ }^{5}$. Grille qui s'avère encore imparfaite dans sa capacité de mesure $^{6}$, mais qui tout de même permet de saisir certains éléments des « arrangements institutionnels » qui, selon la littérature scientifique, fait actuellement la différence entre les régions proactives et celles simple- ment réactives, entre les régions gagnantes et les régions perdantes, entre les milieux innovateurs et ceux qui ne le sont pas.

\section{Éclatement et fragmentation}

Le portrait institutionnel régional contenait à la base, en 1992, 231 organisations publiques, parapubliques et collectives. Ce nombre répertorié a augmenté à 257 organisations en 1996 par la multiplication des corporations de développement local au cours de la première moitié de la décennie 1990. En 2004, nous avons inventorié et classifié 208 de ces organisations qui œuvrent aux trois échelons territoriaux du vaste domaine public régional 02 . Elles exercent plus de 40 fonctions publiques distinctes dans une douzaine de champs d'activités considérés dans le tableau 1. Il existe aussi, dans la région 02, plus de 700 groupes communautaires de diverses natures, généralement ancrés à l'échelle locale.

Tableau 1 - Domaine public Saguenay-Lac-Saint-Jean, 2004

\begin{tabular}{lcccc}
\hline \multirow{2}{*}{\multicolumn{1}{c}{ Secteurs d'activités }} & \multicolumn{5}{c}{ Territoire d'activités } \\
\cline { 2 - 5 } & Local & Supralocal & Régional & Total \\
\hline Affaires municipales & 49 & 4 & 1 & 54 \\
Affaires sociales & 3 & 9 & 5 & 17 \\
Culture et communication & 0 & 0 & 7 & 7 \\
Éducation & 3 & 4 & 7 & 14 \\
Services publics et collectifs & 1 & 1 & 25 & 27 \\
Loisirs et environnement & 0 & 0 & 8 & 8 \\
Commerce & 2 & 0 & 3 & 5 \\
Industrie et construction & 1 & 3 & 15 & 19 \\
Agriculture et alimentation & 1 & 3 & 6 & 10 \\
Travail et main-d'œuvre & 0 & 1 & 5 & 6 \\
Forêts, énergie et ressources & 5 & 0 & 16 & 21 \\
Développement économique & 8 & 4 & 8 & 20 \\
\hline Total & $\mathbf{7 3}$ & $\mathbf{2 9}$ & $\mathbf{1 0 6}$ & $\mathbf{2 0 8}$ \\
Pourcentage par territoire & $\mathbf{3 5 , 1} \%$ & $\mathbf{1 3 , 9} \%$ & $\mathbf{5 1 , 0} \%$ & $\mathbf{1 0 0} \%$ \\
\hline
\end{tabular}

Source : Proulx, M.-U. (dir.) (2004). Profil des organisations publiques, parapubliques et collectives du Saguenay-Lac-Saint-Jean, UQAC.

Nous héritons au Saguenay-Lac-Saint-Jean d'un domaine public éclaté à trois échelons territoriaux et fragmenté au sein de diverses organisations monofonctionnelles qui œuvrent chacune dans leur propre secteur d'activité tel que la santé, l’éduction, l'emploi, la culture, etc. Les municipalités, et aussi les MRC dans une moindre mesure, représentent les seules organisations multifonctionnelles qui demeurent con- 
finées par ailleurs à certains biens et services spécifiques.

\section{La réforme supralocale en cours}

Le fait principal qui a marqué la période récente (1996-2004) concerne l'importante réforme supralocale qui a progressé substantiellement dans sa trajectoire amorcée trois décennies plus tôt. On a alors assisté à des modifications substantielles de certaines organisations publiques qui ont affecté chez celles-ci le nombre, la taille, l'aire de desserte et les modalités de gestion. Furent ainsi éliminées, par fusions ou abolitions, 11 municipalités, 5 organisations du champ des affaires sociales, 5 organisations de celui de l'éducation et 31 organisations du champ du développement économique local. Comme faits saillants à cet effet, notons d'abord la consolidation par fusion des agglomérations urbaines de Saguenay, Alma et Dolbeau ainsi que des villages de Lac-à-la-Croix et de Métabetcouuan ${ }^{7}$. En outre, les quatre territoires MRC furent renforcés. Consolidation et renforcement qui se sont effectués à l'intérieur de grands secteurs publics, soit d'abord le municipal et ensuite le scolaire, la santé et les services sociaux, l'emploi, la formation professionnelle, le développement économique. L'une des composantes oubliées de cette consolidation inachevée concerne le nouveau territoire difforme de la MRC du Fjord-du-Saguenay qui s'avère éclaté par pièces détachées dans la couronne périurbaine et dans la frange périphérique bas-saguenéenne. Notons que les Conseils MRC formés des représentants municipaux furent récemment responsabilisés davantage face aux CLD (centres locaux de développement) de leur territoire qui concentre désormais les efforts de développement local.

Du côté administratif au Saguenay-Lac-Saint-Jean, peu de missions des diverses organisations présentes furent substantiellement modifiées pendant la période observée. Dans le secteur de la santé et des services sociaux, les organisations fusionnées ou intégrées ont même conservé leur mission spécifique à l'intérieur de la mission générale de la nouvelle organisation consolidée. La modification marginale des missions tend vers une bonification à l'égard de la précision des clientèles ciblées, de l'utilisation des outils électroniques, de l'évaluation, de l'évolution, de l'application de normes et d'ouverture sur le monde. La tendance conduit nettement vers la diversification des

produits et des services desservis sur les territoires dans le vaste secteur public régional afin de répondre davantage aux besoins diversifiés des clients. À l'exception des organisations fusionnées par la réforme gouvernementale en région 02 , le territoire de desserte de chacune des 208 organisations observées en 2004 a subi peu de modifications. Nous avons noté par ailleurs un certain nombre de chevauchements de fonctions et de duplications de tâches, surtout entre les différents échelons territoriaux, notamment dans le tourisme et la promotion du développement. Finalement à cette rubrique, le domaine public 02 emploie 20582 ressources humaines en 2004, soit $57 \%$ à l'échelle supralocale, $36 \%$ à l'échelle régionale et 7 \% à l'échelle locale; cet effectif était de 20653 en 1996, dont $9 \%$ à l'échelle locale. Il s'agit d'une expertise publique qui recèle une force considérable à bonifier en région 02 .

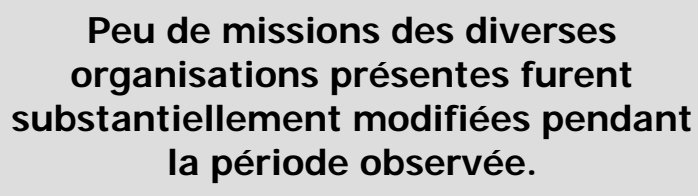

Essoufflement démocratique

Sous l'angle de la démocratie, l'essoufflement souvent diagnostiqué par les médias ${ }^{8}$ apparaît clairement à notre analyse. Signalons que la démocratie territoriale s'avère déjà largement participative grâce aux 180 conseils d'administration actifs dans les 208 organisations désormais présentes au Saguenay-LacSaint-Jean. Il s'agit certes d'un très grand nombre d'instances décisionnelles dont l'agencement général s'avère questionnable globalement sous divers angles, notamment dans son émiettement de l'expertise, ses lourdeurs bureaucratiques, ses corporatismes neutralisants, ses lenteurs décisionnelles. Questionnements à effectuer dans le contexte d'une capacité de mobilisation saturée, généralement surestimée dans son potentiel réel, d'autant plus qu'elle subit actuellement la dévalorisation des non-élus par le gain d'importance des élus sur plusieurs instances décisionnelles, notamment de la CRÉ (Conférence régionale des élus).

Parmi ces 180 CA de la région, $64 \%$ sont ancrés aux échelles locales et supralocales. Si seulement $52 \%$ des représentants sur les CA locaux et supralocaux sont réellement élus par suffrage, ce ratio descend à 
$29 \%$ lorsque nos calculs s'étendent à l'ensemble de la région. Il y a peu d'élus directs aux échelles régionales et MRC. À cet effet, les $71 \%$ de représentants locaux, MRC et régionaux non élus représentent un potentiel de leadership considérable, à harnacher convenablement il va sans dire. D’une manière générale, les collèges électoraux sont devenus un important mécanisme de désignation des conseillers, alors que l'assemblée générale demeure un mécanisme démocratique très utilisé. Soulignons que si seulement 28 organisations publiques (bureaux, directions et autres agences des ministères provinciaux et fédéraux) ne possèdent pas de $\mathrm{CA}$, de nombreux programmes publics souvent importants en ressources sont cependant gérés par celles-ci en étant imputables devant Québec ou Ottawa ${ }^{9}$. Finalement, la démocratie territoriale souffre aussi de l'inégale répartition accentuée du pouvoir décisionnel autant entre les lieux qu'entre les secteurs. En outre, la distance physique entre les acteurs mobilisés dans une vaste région représente une contrainte importante. On note également le peu d'innovations institutionnelles territoriales en regard particulièrement de la nécessaire médiation du pilotage des dossiers collectifs prioritaires et aussi des relations entre les acteurs en général. Il n'y a encore aucun préfet de MRC élu au suffrage dans la région malgré les vertus reconnues de cette formule pour équilibrer le pouvoir politique avec Ville de Saguenay et renforcer le pouvoir régional.

\section{Insuffisance des moyens}

Les ressources financières du vaste domaine public régional 02 s’avèrent certes considérables. Nos données incomplètes, en particulier à propos des ressources allouées par les programmes des gouvernements supérieurs, limitent la comptabilité régionale du budget global. Pour le simple fonctionnement administratif, nous estimons des dépenses publiques régionales globales autour de 1,7 G\$ en 2004. De cette somme estimée, l'impôt foncier municipal totalise près de 420 M\$ en 2004, soit environ $25 \%$ des dépenses publiques régionales affectées au fonctionnement des organisations. Sans compter les divers programmes gouvernementaux exogènes, la dépendance financière de la région à l'égard de Québec et d'Ottawa s'avère évidente.

Il est à signaler par ailleurs qu'au Saguenay, l'important budget de 199 M\$ de la ville principale ne repré- sente que $20 \%$ à $25 \%$ des dépenses publiques de fonctionnement effectuées sur ce territoire, notamment $222 \mathrm{M} \$$ dans la santé et les services sociaux, 366 M\$ dans l'éducation, sans compter la formation professionnelle, la recherche, la $\mathrm{R} \& \mathrm{D}$, le transport, la culture, la Sécurité du revenu et la Sûreté du Québec. À Saint-Félicien, la ville commet un budget de $13 \mathrm{M}$ \$, alors que celui du cégep représente $19 \mathrm{M}$ \$ de dépenses directes. À Roberval, la ville budgétise $12 \mathrm{M}$ \$ et le centre hospitalier en alloue $52 \mathrm{M} \$$. Cette fragmentation budgétaire se fait sentir particulièrement lorsqu'une même fonction publique est exercée par deux ou plusieurs organisations. À titre d'exemple illustratif, la somme de $8 \mathrm{M} \$$ affectée dans la région à l'animation et à la promotion économique en 2004 fut saupoudrée au sein de 35 organisations certes efficaces dans leurs cibles spécifiques, mais néanmoins limitées individuellement en matière d'expertise technique pour assister et soutenir des projets de développement de plus en plus complexes.

\section{La démocratie territoriale souffre aussi de l'inégale répartition accentuée du pouvoir décisionnel autant entre les lieux qu'entre les secteurs.}

Toujours en matière de moyens, il n'existe aucun pouvoir réglementaire et fort peu de pouvoir exécutif à l'échelon régional 02. Beaucoup d'interventions publiques en région sont exogènes, relevant directement de Québec et d'Ottawa. Aussi, la capacité d'interventions endogènes est soumise aux lois, règles et normes des gouvernements supérieurs. Les instances locales, MRC et régionales ont fort peu de contrôle sur l'utilisation des ressources telles que la forêt, les mines, le bassin hydrographique. Les municipalités et les MRC possèdent par contre un pouvoir réglementaire, des ressources financières autonomes et ainsi un pouvoir exécutif considérable. Des zonages sont effectifs pour maîtriser tant bien que mal l'usage du sol. En réalité, le pouvoir d'intervention autonome (à un certain degré) dans la région 02 s'avère très largement local et supralocal. Il est éclaté et fragmenté dans différentes organisations publiques et collectives, dont plusieurs sont totalement indépendantes des conseils municipaux et MRC. Les maires des grandes villes et les préfets de MRC jouent déjà à cet effet un rôle important de médiation et de cohésion, à renforcer éventuellement dans le domaine public à cet échelon. 


\section{Consolidation régionale par la planification}

Depuis les années 1960, la région du Saguenay-LacSaint-Jean a bénéficié de neuf exercices de planification territoriale ${ }^{10}$ effectués en utilisant sept procédures distinctes associées à la théorie de la planification ${ }^{11}$. Celle-ci considère quatre grandes dimensions distinctes (vision - cadre - décisions - interaction) pour effectuer la fameuse liaison entre « connaissance et action ». Liaison qui représente l'essence même de la planification comme pratique de gouverne optimale de l'innovation et du changement à l'échelle d'une collectivité territoriale. Trois formes de pratiques existent à cet effet, soit les planifications allocatrice, radicale et innovatrice. À partir de cette base conceptuelle, les schémas 1 et 2 présentent les quatre dimensions de la planification territoriale qui servent la saisie et la compilation de nos données sur la procédure et les contenus de chacun des neuf exercices effectués en région 02 (tableau 2). Notre analyse par composantes principales ainsi devenue possible nous permet de soulever un certain nombre de constats, de comparer les divers résultats de la planification territoriale et de relativiser la présence de chacune des dimensions.

\section{Schéma 1 - Modèle d'analyse de la planification territoriale}

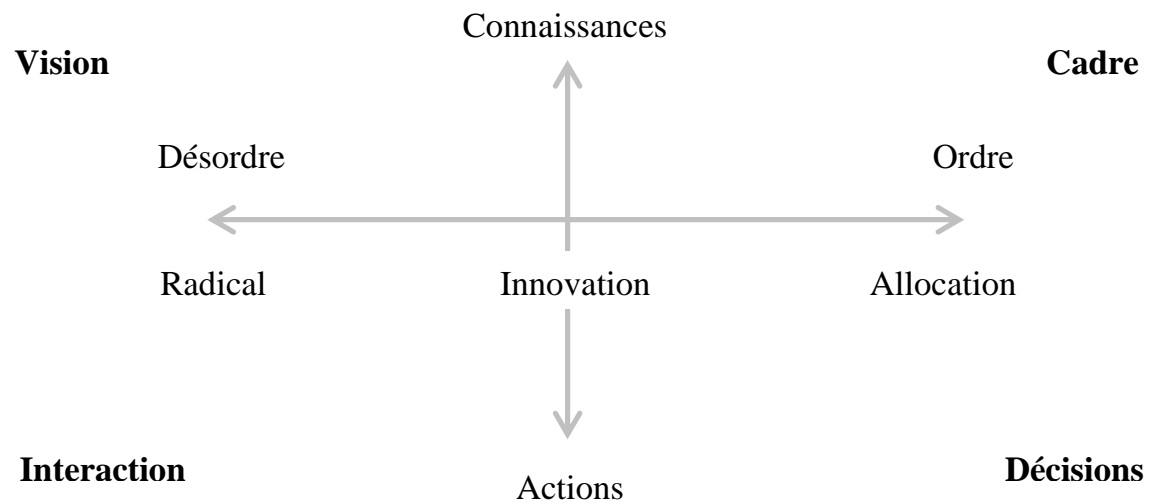

En premier lieu, il apparaît évident que la dimension concernée par la "vision territoriale » a bénéficié d'un contenu variable au fil des exercices. À cet effet, aucune véritable comptabilité régionale réactualisée dans le style «tableau de bord» ne fut formellement établie. Peu de prospective collective ne fut effectuée, si ce n'est à l'exercice P6. Aussi, certains thèmes et secteurs tels que l'énergie, les finances publiques, l'innovation, les institutions, l'apprentissage collectif furent peu scrutés, limitant ainsi la globalité de l'observation visionnaire.

Nous constatons en outre la dominance de la planification de nature stratégique (P2 - P3 - P4 - P7) dont le rigoureux " cadre » associé à l'exercice a certes permis une allocation plus rationnelle des ressources publiques, même si l'évaluation comparative des options stratégiques ne fut que très peu au rendez-vous. Par un concours de circonstances conjoncturelles, trois exercices de planification en région 02 ont utilisé une procédure plus radicale que simplement allocatrice. L'un de ces exercices, relativement informel
(P6), a permis de bonifier considérablement la vision globale élaborée et partagée par les acteurs. Tandis que les deux autres exercices plus formels (P1 et P5) furent largement interactifs et ont à l'évidence fait émerger plusieurs actions territoriales tout à fait innovatrices et structurantes pour la région. Nous y reviendrons.

De fait, notre analyse perçoit fort bien les différences entre les caractéristiques technocratiques et participationnistes, affirmées au cours des différents exercices de planification territoriale. Selon le mélange conjoncturel de ces deux forces, chaque exercice n'aboutit pas aux mêmes résultats. Les exercices plus technocratiques misent largement sur la rationalité dans l'allocation des ressources publiques. Des progrès importants s'avèrent perceptibles Alors qu'une participation élargie fait davantage émerger des initiatives nouvelles, grâce à l'impulsion d'un degré de désordre institutionnel par l'entremise de l'intensification de l'interaction. L'équilibre innovateur entre ces deux forces s'avère évidemment souhaitable sous la forme 
d'actions structurantes, rationnellement faisables selon des critères environnementaux, financiers, so- ciaux, culturels, administratifs, politiques et économiques.

\section{Schéma 2 - Positionnement des exercices de planification territoriale}

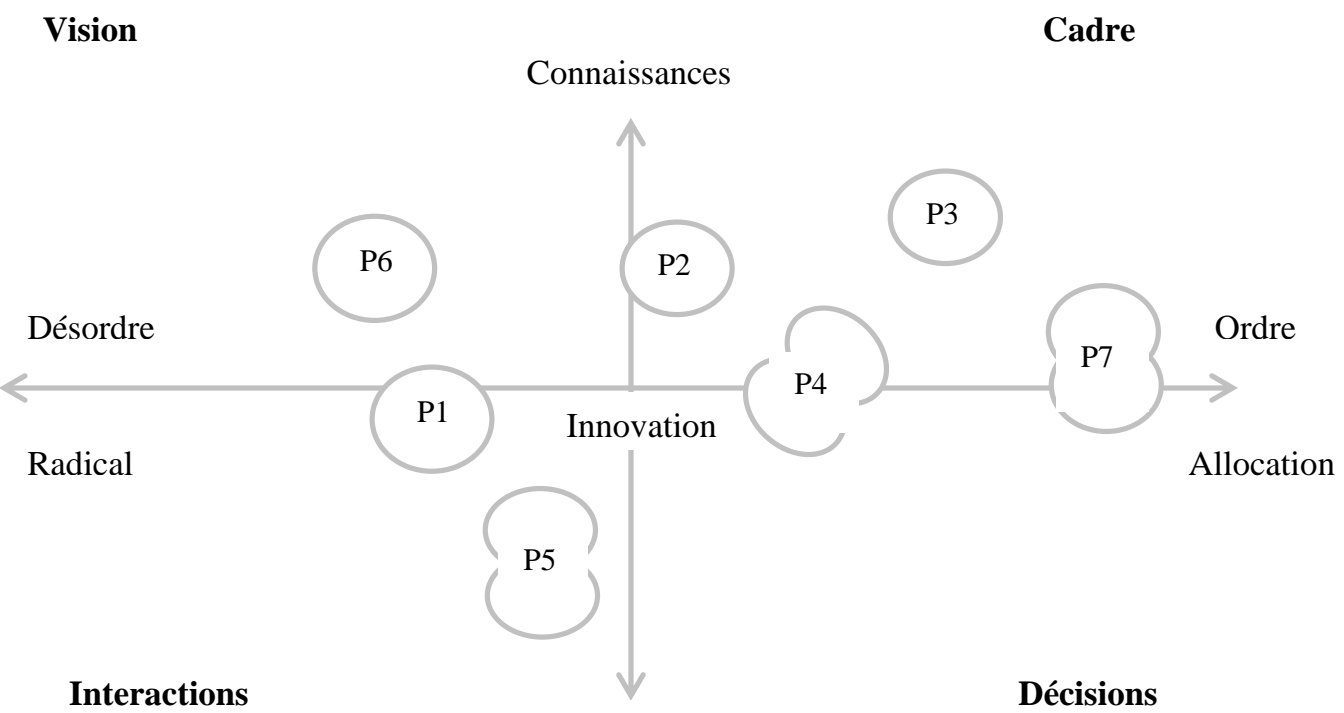

Actions

À cet effet d'actions innovatrices structurantes, la région 02 s'est appropriée certains leviers socioéconomiques, de manière variable, au fil des exercices de planification territoriale. En réalité, des acquis importants tels que des équipements de prestige (Pulperie, Grands Jardins, Véloroute, réseau muséal, centrales et réseaux hydroélectriques, éléments patrimoniaux, centres de R\&D), des organisations novatrices (coopératives, SOCCRENT, RLDD, STASS, CREPAS, conseils, alliances, associations...) et des instruments collectifs (fiscalité, règlements, incubateurs, parcs, services d'accueils, etc.) furent au rendez-vous dans la région. Force est de constater cependant les limites institutionnelles de cette appropriation territoriale de leviers originaux de développement soutenue par le domaine public. Des faiblesses et des manques régionaux évidents apparaissent à notre lecture.

\section{Le contrôle de l'utilisation des ressources, notamment le sol, s'avère insuffisant.}

Soulignons d'abord qu'après quatre décennies de planification ${ }^{12}$, la région 02 balbutie encore dans la formulation d'une véritable vocation régionale : Domaine du Roy, Oasis nordique, Porte d'entrée du Nord, Laboratoire de développement durable, Pays des bleuets, Vallée de l'aluminium, Royaume du bois, etc. Il n'y a pas une unique référence collective, stable et continue, pouvant être évoquée symboliquement et servir de "projet territorial mobilisateur " pour les acteurs sur le terrain. Le nécessaire repositionnement actuel de l'image territoriale rend impérative l'affirmation vigoureuse de cette « vocation » par un marketing territorial approprié. Or, ladite planification stratégique de la décennie 1990 n’a paradoxalement pas conduit à ce résultat, dont l'atteinte a tout de même progressé de manière significative avec la récente désignation de "créneaux régionaux d'excellence » par l'entremise de la politique industrielle de Québec. Aussi, la région ne possède pas de fonds autonome substantiel de développement. Le contrôle de l'utilisation des ressources, notamment le sol, s'avère insuffisant, notamment en zones de villégiature et périurbaines. Bien que la problématique de la forêt fasse récemment l'objet d'une réflexion intensive tout à fait bienvenue, la région ne bénéficie pas de dossier formel sur certains enjeux cruciaux tels que le bassin hydroélectrique, les terres en friche, le potentiel éolien, l'occupation nordique, les TIC ${ }^{13}$ et les autres moteur du futur développement régional. La région ne possède pas de mécanismes professionnels pour établir systématiquement la faisabilité multicritère des projets collectifs structurants qu'elle nécessite pour progresser. Elle ne possède pas non plus de mécanisme formel de dépistage de promoteurs, d'entrepre- 
neurs, de leaders et de porteurs de dossiers. Cette région manque, en outre, de mécanismes pour faciliter les interfaces adéquates, notamment pour réguler les relations patronales-syndicales, les relations entre Ville de Saguenay et ses partenaires municipaux et non municipaux, les relations entre les divers lieux, les divers milieux, les divers secteurs. Il y a en somme encore peu d'alliances, d'ententes et de partena- riats interorganisationnels. Mis à part les infrastructures de transport dont la demande sociale actuelle s’avère globalement énorme, le développement local et régional endogène illustre clairement un manque de projets d'actions structurantes, une absence de projet moteur. À l'évidence de notre lecture institutionnelle, on se doit de questionner le pilotage collectif du développement dans la région 02 par la planification.

\section{Tableau 2 - Le contenu des exercices de planification régionale au Saguenay-Lac-Saint-Jean}

\begin{tabular}{|c|c|c|c|c|c|c|c|c|c|}
\hline & P1 & $\mathbf{P} 2$ & P3 & P4 & P5 & P6 & P5 & P7 & P7 \\
\hline Procédures & $\begin{array}{c}\text { Enquête- } \\
\text { participation }\end{array}$ & Mission & $\begin{array}{l}\text { Schéma } \\
\text { régional }\end{array}$ & $\begin{array}{l}\text { Schéma } \\
\text { MRC }\end{array}$ & CSÉR I & $\begin{array}{l}\text { Congrès } \\
\text { éthique }\end{array}$ & CSÉR II & PSR I & PSR II \\
\hline DIMENSIONS & 1961-1965 & 1969 & 1977 & 1982-1990 & 1983-1984 & 1988-1990 & 1990-1991 & 1996 & 2001 \\
\hline Vision & $\begin{array}{l}\text { Consultation } \\
\text { élargie }\end{array}$ & $\begin{array}{l}\text { Réflexion } \\
\text { collective } \\
\text { bien } \\
\text { articulée }\end{array}$ & $\begin{array}{l}\text { Plusieurs } \\
\text { études } \\
\text { d'experts }\end{array}$ & $\begin{array}{l}\text { Docu- } \\
\text { ments } \\
\text { Études } \\
\text { Consulta- } \\
\text { tions }\end{array}$ & $\begin{array}{l}\text { Portrait } \\
\text { global } \\
\text { exhaustif }\end{array}$ & $\begin{array}{l}\text { Réflexion } \\
\text { collective } \\
\text { éthique sur } \\
\text { les finalités }\end{array}$ & Synthèse & $\begin{array}{l}\text { Portrait } \\
\text { régional } \\
\text { Grandes } \\
\text { tendances }\end{array}$ & $\begin{array}{l}\text { Grandes } \\
\text { tendances }\end{array}$ \\
\hline $\begin{array}{l}\text { Cadre } \\
\text { stratégique }\end{array}$ & $\begin{array}{l}3 \text { stratégies : } \\
\text { Bleuets } \\
\text { Scieries } \\
\text { Emplois }\end{array}$ & $\begin{array}{l}\text { Grands } \\
\text { objectifs } \\
\text { Pôle de } \\
\text { croissance } \\
\text { saguenéen } \\
\text { Parcs } \\
\text { régionaux } \\
\text { Transfor- } \\
\text { mation } \\
\text { Institu- } \\
\text { tions } \\
\text { régionales }\end{array}$ & $\begin{array}{l}5 \text { grandes } \\
\text { stratégies }\end{array}$ & $\begin{array}{l}\text { Grandes } \\
\text { orientation } \\
\text { d'aména- } \\
\text { gement }\end{array}$ & $\begin{array}{l}\text { Nouvelles } \\
\text { orienta- } \\
\text { tions } \\
\text { régionales }\end{array}$ & $\begin{array}{l}8 \text { grandes } \\
\text { finalités }\end{array}$ & $\begin{array}{l}\text { Orienta- } \\
\text { tions }\end{array}$ & $\begin{array}{l}\text { Plusieurs } \\
\text { stratégies }\end{array}$ & $\begin{array}{l}\text { Stratégies } \\
\text { plus } \\
\text { précises } \\
\text { Créneaux } \\
\text { Enjeux }\end{array}$ \\
\hline Actions & $\begin{array}{l}\text { Proposition } \\
\text { de } 5 \text { unités } \\
\text { d'aménage- } \\
\text { ment du } \\
\text { territoire }\end{array}$ & Peu & Peu & $\begin{array}{l}\text { Équipe- } \\
\text { ments } \\
\text { Infrastruc- } \\
\text { tures } \\
\text { ciblées }\end{array}$ & $\begin{array}{l}661 \text { projets } \\
\text { proposés } \\
87 \text { actions } \\
\text { priorisées } \\
\text { STASS } \\
\text { SOCCRENT } \\
\text { R\&d } \\
\text { Autoroute } \\
70 \\
\text { Relations } \\
\text { syndicales- } \\
\text { patronales }\end{array}$ & $\begin{array}{l}\text { Forum } \\
\text { permanent }\end{array}$ & $\begin{array}{l}\text { Consoli- } \\
\text { dation des } \\
\text { acquis } \\
\text { SOCCRENT } \\
\text { CQRDA } \\
\text { RLDD }\end{array}$ & $\begin{array}{l}\text { Consolida- } \\
\text { tion des } \\
\text { acquis } \\
\text { Grands } \\
\text { Jardins } \\
\text { Piste } \\
\text { cyclable } \\
\text { CREPAS }\end{array}$ & $\begin{array}{l}\text { Vallée de } \\
\text { l'aluminium } \\
\text { CREPAS } \\
\text { Tableau de } \\
\text { bord } \\
\text { Fonds } \\
\text { «forêt » }\end{array}$ \\
\hline Interaction & $\begin{array}{l}1500 \text { familles } \\
\text { mobilisées } \\
30 \text { municipa- } \\
\text { lités impli- } \\
\text { quées } \\
\text { CER } \\
\text { Collaboration } \\
\text { verticale }\end{array}$ & $\begin{array}{l}\text { CAR } \\
15 \text { minis- } \\
\text { tères }\end{array}$ & $\begin{array}{l}\text { Conseils } \\
\text { régionaux } \\
\text { sectoriels }\end{array}$ & $\begin{array}{l}\text { Comités } \\
\text { Participa- } \\
\text { tion de la } \\
\text { société } \\
\text { civile } \\
\text { Concer- } \\
\text { tation }\end{array}$ & $\begin{array}{l}\text { Concerta- } \\
\text { tion } \\
\text { Près } 2000 \\
\text { acteurs } \\
\text { mobilisés } \\
\text { Sommet }\end{array}$ & $\begin{array}{l}\text { Ateliers } \\
\text { animés } \\
\text { d'un } \\
\text { congrès } \\
\text { progressif } \\
\text { sur } 2 \text { ans }\end{array}$ & $\begin{array}{l}12 \text { tables } \\
\text { sectoriel- } \\
\text { les } \\
\text { Sommet }\end{array}$ & $\begin{array}{l}\text { Comité } \\
\text { aviseur } \\
\text { Commis- } \\
\text { sions } \\
\text { sectoriel- } \\
\text { les } \\
\text { Forum } \\
\text { régional } \\
\text { États } \\
\text { généraux } \\
\text { Ententes- } \\
\text { cadres }\end{array}$ & $\begin{array}{l}\text { Commis- } \\
\text { sions } \\
\text { sectoriel- } \\
\text { les } \\
\text { Ententes- } \\
\text { cadres } \\
\text { Rendez- } \\
\text { vous des } \\
\text { régions }\end{array}$ \\
\hline
\end{tabular}


Nous constatons à cet effet, la dominance croissante des exercices de planification strictement sectorielle (éducation, santé et services sociaux, transport, culture, tourisme...). Domination et replis sectoriels qui se renforcent actuellement avec la planification régionale dans les créneaux d'excellence (aluminium, agroalimentaire, forêt, tourisme d'aventure...). Mis à part les schémas d'aménagement qui possèdent une envergure globale, l'échelon MRC possède aussi désormais plusieurs plans strictement sectoriels concernés par le développement, l'emploi, l'éducation, les services communautaires, la gestion de services spécialisés. Alors qu'à l'échelle locale, les plans d'urbanisme, de transport en commun, de voirie, de loisirs, de divers équipements généralisent cette division par secteurs de la réflexion collective sur l'action. Secteurs désormais organisés, qui ont tendance à se replier sur leur propre perspective d'observation de la réalité régionale.

Tant et si bien que la planification régionale 02 respecte de moins en moins le principe de la globalité si essentiel. Certes bien confectionnés et très utiles, les plans détachés par secteurs et par territoires ont désormais préséance sur la globalité. Selon notre analyse, la région du Saguenay-Lac-Saint-Jean apparaît globalement plus divisée que jamais par morceaux sectoriels et territoriaux. En outre, la séparation de plus en plus claire entre les dimensions "cadre stratégique » et « décisions opérationnelles » par des procédures distinctes de planification accentue la disjonction entre les planificateurs. Bref, nous avançons que la région nécessite un nouvel exercice de planification régionale globale de forme innovatrice, capable de faire émerger de nombreuses actions structurantes. La crise économique et sociale régionale sans précédent qui est vécue depuis 2003 rend, à notre avis, cette planification tout à fait impérative.

\section{Conclusion}

Selon notre lecture, le contexte institutionnel du Saguenay-Lac-Saint-Jean n'est actuellement pas optimal pour affronter la restructuration économique et sociale qui nécessite des actions innovatrices et vigoureuses. Le domaine public fragmenté et éclaté, l'essoufflement démocratique, le manque de moyens ainsi qu'une planification territoriale inadéquate contraignent très lourdement la proactivité et l'innovation pourtant impératives dans le nouveau contexte continental et mondial en changement continue.

\section{Nous avançons que la région nécessite un nouvel exercice de planification régionale globale de forme innovatrice.}

\section{Schéma 3 - Prochaine procédure de planification régionale}

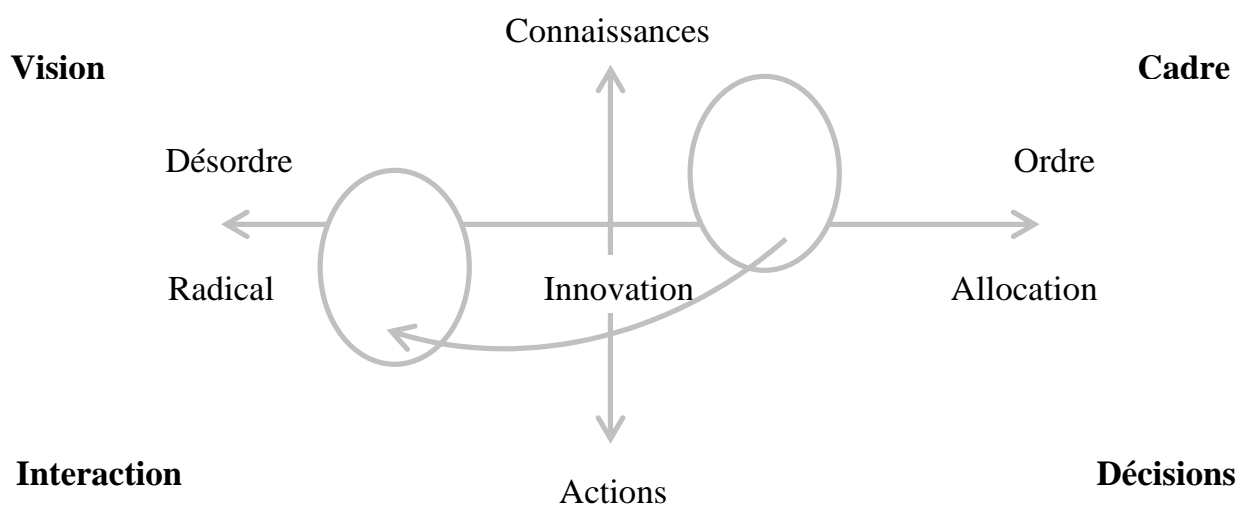

À cet effet, il nous apparaît tout à fait indiqué, dans cette région 02, d'interpeller selon une vision globale les multiples acteurs hors de leur repli corporatiste réciproque, confortés par un cadre stratégique sectoriel bien à eux, mais que pour eux. Il devient urgent de désenclaver les nombreux plans par morceaux sec- toriels et territoriaux, tout en conservant leurs importants apports réels et utiles. Nous proposons à cet effet de mettre en œuvre une planification globale de nature radicale afin d'atteindre un équilibre pleinement innovateur en matière d'actions structurantes. Pour ce faire, les dimensions « vision » et « interaction » doivent 
être interpellées impérativement par les planificateurs sectoriels, locaux, MRC et régionaux.

Plusieurs enjeux s'offrent alors à la réflexion collective, notamment la poursuite de la consolidation supralocale, le renforcement démocratique, la dotation de moyens financiers et réglementaires supplémentaires, l'élaboration de dossiers techniques (forêt, éolien, TIC, terres en friche, créneaux post-industriels...), la mise en œuvre de nouveaux outils de développement, la cohérence globale du domaine public régional, etc. Ces enjeux doivent, à notre avis, être interpellés dans un esprit d'apprentissage collectif et d'innovation territoriale.

\section{Notes et références}

1 L'auteur est Docteur en économie régionale et professeur titulaire au département des sciences économiques et administratives. Ses recherches portent sur la dynamique spatiale et la théorie du développement territorial.

2 Il est à noter qu'une autre catégorie de territoires est actuellement sur la planche à dessin avec la désignation de zones spécialisées appelées les « créneaux d'excellence ».

3 Territoire 02 découpé dans le Royaume historique de Saguenay afin d'offrir une aire administrative homogène.

4 Une analyse de la turbulence régionale vécue lors des innondations massives de 1996 au Saguenay-Lac-SaintJean fut effectuée dans Proulx, M.-U. (dir.) (1998), Une région dans la turbulence, Sillery, PUQ. Nous considérons que les difficultés économiques de 2003-2004-2005 engendrent un chaos institutionnel supérieur à celui de 19961997.
5 Texte rédigé sur la base des Profils 1992-1996-2004 des Organisations publiques, parapubliques et collectives $d u$ Saguenay-Lac-Saint-Jean. Voir aussi « De la vision en planification territoriale» sur le portail du mouvement Vision Saguenay 2025.

$6 \quad$ Nous saisissons encore très mal des éléments ou propriétés institutionnels tels que les processus identitaires, la mobilisation sociale, la circulation de l'information, la solidarité communautaire, etc.

7 Dans cette région, la consolidation villages-paroisses tant désirées au Québec était déjà acquise grâce à l'élimination d'une quarantaine de petites municipalités et de dizaines de commissions scolaires, entre 1961 et 1996.

8 Postes électifs ou désignés non comblés; élus par acclamation; scrutins électoraux boudés; inégale répartition du pouvoir; syndrome des TLM (toujours les mêmes); potentats locaux et régionaux.

9 En 2004, le bureau régional de Développement Économique Canada a alloué près de $15 \mathrm{M} \$$ sans conseil d'administration, alors que la CRÉ associée au vrai pouvoir régional n'a obtenu qu'un budget de 1,5 M\$.

10 Les plans locaux, les plans d'urbanisme, les plans sectoriels et les plans d'action spécifiques ne furent pas considérés ici.

11 Voir Proulx, M.-U. (1996). «Trois décennies de planification régionale au Québec », dans Proulx, M.-U. (dir.) Le phénomène régional au Québec, Sillery, PUQ.

12 Voir Proulx, M.-U. « Quatre décennies de planification territoriale au Québec », communication livrée au Congrès ACFAS 2005, UQAC, à paraître.

13 Technologies d'information et de communication. 


\section{Formulaire d'abonnement}

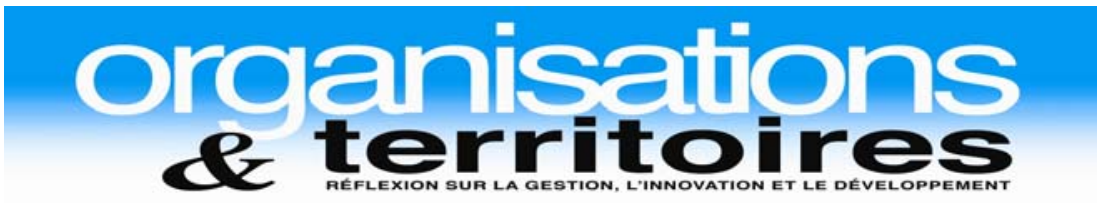

JE DÉSIRE M'ABONNER À LA REVUE ORGANISATIONS ET TERRITOIRES ET JE JOINS UN CHÈQUE AU MONTANT DE : $\$$

Nouvel ABonné : $\square$ RENOUVELlEMENT : $\square$ DATE :

Abonnement institutionnel (6 numéros) $\quad 103,52 \$$ (Toutes taxes incluses)

Abonnement individuel (6 numéros) 66,71 \$ (Toutes taxes incluses)

Abonnement hors Canada (6 numéros) 145,00 \$

* Tous les abonnements sont d'une durée de deux ans.

- Je désire que mon abonnement débute avec le DERNIER numéro paru.

- Je désire que mon abonnement débute avec le PROCHAIN numéro à paraître.

Faites-nous parvenir vos coordonnées :

Nom :

Organisation :

Adresse :

Téléphone :

Télécopieur :

Courriel :

\section{ADRESSE D’EXPÉDITION}

Revue Organisations et territoires

Université du Québec à Chicoutimi

555, boulevard de l’Université, bureau H5-1040

Chicoutimi (Québec) CANADA G7H 2B1

Téléphone : (418) 545-5011, poste 4530 Télécopieur : (418) 693-9072

Courriel : revue_ot@uqac.ca Site Web : www.uqac.ca/revueot 